\title{
Changes in pigment, spectral transmission and element content of pink chicken eggshells with different pigment intensity during incubation
}

Yue Yu, Zhanming Li, Jinming Pan

Objective: The objective of this study was to investigate changes in pigment, spectral transmission and element content of chicken eggshells with different intensities of pink pigment during incubation period. We also investigated the effects of the region (small, equator and large pole) and pink pigment intensity of the chicken eggshell on the percent transmittance of light passing through the chicken eggshells.

Method: Eggs of comparable weight from a meat-type breeder (Meihuang) were used, divided based on three levels of pink pigment (light, medium and dark) in the eggshells. During the incubation (0-21 d), the values of the eggshell pigment $\left(\Delta E, L^{*}, a^{*}, b^{*}\right)$ and the percent transmittance of light passing through the eggshell were measured.

Result: Three measured indicators of eggshell color, $\Delta E, L^{*}$ and $a^{*}$, did not change significantly during incubation. Using the visible wavelength range of $380-780 \mathrm{~nm}$, the percent transmittance of light for different regions and intensities of eggshell pigmentation was measured. Compared with other regions and pigment intensities, eggshell at the small pole and with light pigmentation intensity showed a highest percent transmittance of light. And the transmittance value varied significantly $(P<0.001)$ with incubation time. The element analysis of the eggshells with the different levels of pink pigment showed that the potassium content of the eggshells for all pigment levels decreased significantly during the incubation.

Conclusion: In summary, the pigment intensity and the region of the eggshell influenced the PT of the eggshell. Differences in the spectral characteristics of different eggshells may influence the effects of photostimulation during the incubation of eggs. All of these results will be applicable for perfecting the design of light intensity for lighted incubation to improve productivity. 

with different pigment intensity during incubation

4 Yue Yu ${ }^{\mathrm{a}, \mathrm{b}}$, Zhanming $\mathrm{Li}^{\mathrm{a}}$, Jinming Pan ${ }^{\mathrm{a}, \mathrm{b}^{*}}$

5

${ }^{a}$ Department of Biosystems Engineering, Zhejiang University, Hangzhou 310058, China

$7 \quad$ State Key Laboratory of Soil Plant Machinery System Technology, Beijing 100083, China

11 *Correspondence to: Dr. Jinming Pan, Department of Biosystems Engineering, Zhejiang

12 University, Hangzhou 310058, China. E-mail: panhouse@zju.edu.cn 


\section{Abstract}

15 Objective: The objective of this study was to investigate changes in pigment, spectral transmission and element content of chicken eggshells with different intensities of pink pigment during the incubation period. We also investigated the effects of the region (small pole, equator and large pole) and pink pigment intensity of the chicken eggshell on the percent transmission of light passing through the chicken eggshells.

Method: Eggs of comparable weight from a meat-type breeder (Meihuang) were used, and divided based on three levels of pink pigment (light, medium and dark) in the eggshells. During the incubation $(0-21 \mathrm{~d})$, the values of the eggshell pigment $\left(\Delta \mathrm{E}, \mathrm{L}^{*}, \mathrm{a}^{*}, \mathrm{~b}^{*}\right)$ were measured. The percent transmission of light for different regions and intensities of eggshell pigmentation was measured by using the visible wavelength range of 380-780 $\mathrm{nm}$.

Result: Three measured indicators of eggshell color, $\Delta \mathrm{E}, \mathrm{L}^{*}$ and $\mathrm{a}^{*}$, did not change significantly during incubation. Compared with other regions and pigment intensities, eggshell at the small pole and with light pigmentation intensity showed the highest percent transmission of light. The transmission value varied significantly $(P<0.001)$ with incubation time. The element analysis of eggshells with different levels of pink pigment showed that the potassium content of the eggshells for all pigment levels decreased significantly during incubation.

Conclusion: In summary, pigment intensity and the region of the eggshell influenced the percent transmission of light of eggshell. Differences in the spectral characteristics of different eggshells may influence the effects of photostimulation during the incubation of eggs. All of these results will be applicable for perfecting the design of light intensity for lighted incubation to improve 
35 productivity. 


\section{Introduction}

During the artificial incubation of chicken eggs, five factors are well known to play important roles in embryonic development and are usually carefully controlled: temperature, humidity, partial pressures of oxygen and carbon dioxide (ventilation), and the frequency of turning eggs (Nelson et al., 2004, Portugal et al., 2014). Moreover, during natural incubation in birds, eggs would certainly receive light stimulation when birds leave for feeding, whereas in commercial incubation, complete darkness is often employed. In recent years, studies have shown that embryonic development is affected by light (Adam and Dimond, 1971, Rogers, 2008, Shafey and Al-Mohsen, 2002). Photostimulation during incubation can improve growth and hatchability (Garwood et al., 1973, Shafey and Al-Mohsen, 2002, Walter and Voitle, 1972) and decrease incubation time (Fairchild and Christensen, 2000, Ghatpande et al., 1994, Shafey et al., 2002), thereby increasing productivity. In contrast, some reports have also indicated that photostimulation during incubation reduced or did not affect hatchability (Archer and Mench, 2014, Archer et al., 2009, Özkan et al., 2012a). Taminmie and Fox (1967) reported a delay in hatchability and increased incidence of embryonic abnormalities in those chicks exposed to light during incubation. However, some reports found no effect on hatchability when eggs were exposed to light during incubation (Lauber and Shutze, 1964, Zakaria, 1989).

53 These discrepancies might be caused by the spectral characteristics of light that reaches the embryos during incubation. Eggshell pigments differ by species and include white, pink, brown and green, and even the eggs laid by the same species can have different intensities of eggshell pigmentation (Moreno and Osorno, 2003). Comparison of untreated and manually pigmented 
57 eggshells indicated that the pigmentation of eggshells influenced the spectral transmission of

58 light into the egg (Shafey et al., 2002). Thus, the effect of light on incubation depends on the

59 characteristics of eggs, especially of eggshells. In fact, the photostimulation effect on embryonic

60 development depends on the amount of light that reaches the embryos (Ghatpande et al., 1994).

61 The spectral transmission of light is influenced by the pigment and thickness of the eggshell

62 (Bameliset al., 2002, Maurer et al., 2011, Shafey et al., 2005). Unfortunately, this information is

63 still neglected during egg incubation.

Thus, the objective of this research was to investigate changes in pigment intensity, spectral transmission and element content of pink chicken eggshells with different pigment intensities during incubation. The effects of the region (small pole, equator pole and large pole) of the eggshells were also studied. The change of pigment, element content and percent transmission

\section{Materials and methods}

All experimental protocols were approved by the committee of the Care and Use of Animals

of Zhejiang University. The methods were carried out in strict accordance with the guidelines of the Association for the Study of Animal Behaviour Use of Zhejiang University.

\section{Grouping of eggs by eggshell pigment}

Three hundred freshly laid pink fertilized eggs with approximately comparable weights, 
eggs were distributed among three groups according to the pigment intensity of the pink eggshell, i.e., light intensity pigment (LIP), medium intensity pigment (MIP) and dark intensity pigment (DIP). The pigment intensity $\left(\Delta \mathrm{E}, \mathrm{L}^{*}, \mathrm{a}^{*}\right.$ and $\left.\mathrm{b}^{*}\right)$ was measured with a color tester $(\mathrm{CR}-400$, Konica Minolta, Tokyo, Japan). Three regions (small pole, equator and large pole) were measured, and the average of the values of the three regions was used for whole-egg analyses. $\Delta \mathrm{E}$ represents CIE $\Delta \mathrm{E}$ colour distance between the standard white plate and the sample; $\mathrm{L}^{*}$ represents luminance or brightness of sample; $a^{*}$ and $b^{*}$ represent colour values along the redgreen and yellow-blue opponent axes, respectively. The value for $\Delta \mathrm{E}=\left(\Delta \mathrm{a}^{2}+\Delta \mathrm{b}^{2}+\Delta \mathrm{L}^{2}\right)^{1 / 2}$ was used $\Delta \mathrm{E}$ as the standard of the classification basis, and according to the value, we defined the value of $\Delta \mathrm{E}=11.72 \sim 22.49$ as the LIP, $\Delta \mathrm{E}=22.54 \sim 28.16$ as the MIP, $\Delta \mathrm{E}=28.17 \sim 41.38$ as the DIP. All eggs except the samples for 0 days were fumigated with formaldehyde solution and were numbered before incubation.

\section{Incubation}

The three groups of eggs were placed into their respective tray and then in a commercial incubator (EI-hatching, Qingdao Xingyi, Qingdao, China). The internal dimensions of the incubator were $100 \mathrm{~cm}$ length $\times 110 \mathrm{~cm}$ width $\times 95 \mathrm{~cm}$ height. The incubator was calibrated using a standard thermometer and hygrometer before hatching the experimental eggs. The incubator was automatically maintained at $38.0 \pm 0.1{ }^{\circ} \mathrm{C}$ and $60 \pm 1 \%$ relative humidity (RH) during the entire incubation. The turning time interval during incubation was three hours.

\section{Sampling and measurement}

On days $0,4,8,12,16$ and 21 during the incubation, 10 eggs were randomly sampled from 
99

100

101

102

103

104

105

106

107

108

109

each of the three trays for measurements of the chrominance difference, spectral transmission and element content of eggshells. First, the pigment intensity of three regions (small pole, equator and large pole) of each egg was measured. Then, the eggs were carefully broken, and the eggshells were washed with water and dried with paper towels. Three pieces $\left(1\right.$ to $\left.1.5 \mathrm{~cm}^{2}\right)$ with the membrane intact were separated from the small pole, equator and large pole of each eggshell. The light transmission of each piece was measured using a spectrometer (QE6500, Ocean Optics, Dunedin, FL, USA). The effective detecting area was $0.25 \mathrm{~cm}^{2}$. The light source was a halogen lamp (HL-2000-LVP-HP 24 V, Ocean Optics, Dunedin, FL). Spectral transmission of the eggshell was recorded over the wavelength range of 380 to $780 \mathrm{~nm}$ (visible range). Figure 1 shows a schematic of the detection equipment for PT. After that, the eggshell thickness of three regions (small pole, equator and large pole) was also measured. Pieces of three regions abovementioned were measured three times respectively to get a more accurate average value.

Finally, the eggshell samples were analyzed for potassium (K), sodium (Na), phosphorus (P), calcium (Ca) and magnesium (Mg) using an ICP-MS (ELAN DRC-e, PerkinElmer) (Ionov, Savoyant, \&Dupuy, 1992). The eggshells were dried at $50{ }^{\circ} \mathrm{C}$ and then crushed with a pulverizer (Q-250B, Qijian, China) for 1 min to obtain homogeneous samples. For each group, six samples per sampling were selected for the chemical analyses; of those six samples, pairs were mixed so that there were three replicates for each sampling of each group.

\section{Statistical analysis}

A one-way ANOVA model (SPSS 17.0) was used to test for differences among the different levels of pigment intensity and among the regions of the eggshells during incubation. Reported 
120

121

122

123

124

125

126

127

128

129

130

131

132

133

134

135

136

137

138

139

140

values represent the mean \pm standard error, and the level of significance was taken as $P<0.05$.

When significance was detected, the differences between the treatment means were tested using the least significant difference (LSD) procedure.

\section{Results}

\section{Changes in eggshell pigment during incubation}

The change in the $\Delta \mathrm{E}, \mathrm{L}^{*}, \mathrm{a}^{*}$ and $\mathrm{b}^{*}$ values of pink pigmented eggshells during the incubation was evaluated. Significant changes were observed in the $b^{*}$ value of pink pigment eggshells during incubation (Table 1). The value of $b^{*}$ was significantly reduced at day 4 compared with those of day 0 . The value $\mathrm{f} \mathrm{b}^{*}$ represents the yellow and blue balance of the sample, with increases and decreases in the value representing more yellow and blue, respectively. However, the results of Table 1 showed no significant changes in the $\Delta \mathrm{E}, \mathrm{L}^{*}$ or $\mathrm{a}^{*}$ values of pink pigmented eggshells.

\section{Changes in the PT according to different regions and different eggshell pigment intensities}

\section{during incubation}

We measured the change in PT of light over the visible range at the small pole, the equator and the large pole of the eggshells on days $0,4,8,12,16$ and 21 during incubation. The results of the whole period were consistent. Using day 0 (Figure 2) as an example, the PT value of the small pole was the highest and was significantly different from those of the other regions $(P<$ 0.05). The values at the equator and the large pole were $8.18 \%$ and $6.39 \%$ lower, respectively, than that of the small pole. Although the PT of the large pole was slightly higher than the equator, there was no significant difference between the large pole and the equator. The pattern of PT 
141 values at the three locations remained consistent during the entire incubation. Throughout 142 incubation, the highest PT was observed in the small pole, followed by the equator and the large 143 pole.

144 We also measured the change in PT of light over the visible range for the LIP, MIP and DIP 145 eggshells during the entire incubation. Again using day 0 (Figure 3) as an example, the LIP 146 showed the highest PT of the three colour groups, and there was a significant difference among 147 the colors $(P<0.05)$. The PT of LIP was $5.11 \%$ and $2.32 \%$ higher than DIP and MIP, respectively. The PT value for MIP was not significantly different from the other conditions.

Further, the difference in PT values for the three colors of eggshells during the incubation period was the same as on day 0. The highest PT was observed in LIP eggshells, followed by MIP and then DIP eggshells. However, although the PT of MIP eggshells was higher than that of DIP eggshells, the only significant difference $(P<0.05)$ observed was between the PT values of LIP and DIP.

Figure 4 shows that the incubation could be separated into two periods, days 0-16 and days 16-21, according to the PT of the eggshell. The PT of eggshells increased gradually, to $13.53 \%$ on day 16 and rapidly increased to $24.12 \%$ on day 21 . The trend over the whole period (0-21 day) was significant (Figure $4, P<0.001$ ). And Figure 4 shows that the PT of the eggshell was also significant decreased from day 0 to day 16 (Figure $4, P<0.001$ ).

\section{Changes in thickness of eggshell during incubation}

Eggshell thickness was significantly decreased during the incubation $(P<0.01)$ (Figure5).

The value of thickness on day $0(0.35 \mathrm{~mm})$ was significantly different from day $16(0.331 \mathrm{~mm}$, 
162 163

$P<0.01)$ and day $21(0.327 \mathrm{~mm}, P<0.001)$, and slight change was observed on day $4(0.342 \mathrm{~mm})$, day $8(0.339 \mathrm{~mm})$ and day $12(0.337 \mathrm{~mm})(P>0.05)$. These data indicated that the significant decrease of eggshell thickness appeared on day 16. Theoretically speaking, the decrease of eggshell thickness may improve the PT.

\section{Changes in the element content of the eggshell during incubation}

The concentrations of various elements, during the incubation, in eggshells with different intensities of pigment are shown in Table 3 . The $\mathrm{Ca}$ and $\mathrm{P}$ contents of eggshells with different intensities of pigment did not change significantly during the incubation. However, the $\mathrm{Na}$ content of the MIP decreased significantly $(P<0.05)$ during the incubation, and the $\mathrm{K}$ content of the eggshells with all levels of pigment intensity decreased significantly during the incubation (LIP: $P<0.05$, MIP: $P<0.01$, DIP: $P<0.05$ ).

\section{Discussion}

Our results clearly demonstrate that the intensity of the pigment and the region of the eggshell influenced the spectral characteristics of eggshells, and these results were consistent throughout the incubation. In addition, the PT and the potassium content of the eggshells decreased as the intensity of the pink pigment increased.

Light has a profound effect on embryonic growth (Özkan et al., 2012a, Özkan et al., 2012b, Shafey, 2004, Shafey and Al-Mohsen, 2002, Shafey et al., 2005, Zhang et al., 2012). However, little information is available on the factors that cause the various changes in response to photostimulation during incubation (Archer and Mench, 2014, Rozenboimet al., 2003). When different intensities of fluorescent light were used during the incubation of eggs, the acceleration 
183

184

185

186

187

188

189

190

191

192

193

194

195

196

197

198

199

200

201

202

of embryonic development was dependent on the quantity of light that reached the embryos

(Ghatpande et al., 1994, Gold and Kalb, 1976, Tamimie and Fox, 1967). When pigmented and

unpigmented Japanese quail eggs were placed in the same light environment during incubation,

the embryonic development of the pigmented eggs was slower than that of the unpigmented eggs,

which hatched earlier (Coleman and McNabb, 1975).

Meihuang birds lay pink eggs with a wide variety in the intensity of the color of the eggshell. The main pigment of pink eggshells is protoporphyrin, and the color of the pigment depends on the selective absorption of certain wavelengths of light and the reflection of others. In this study, three measured indicators of eggshell color, $\Delta \mathrm{E}, \mathrm{L}^{*}$, and $\mathrm{a}^{*}$ did not change significantly during the incubation. However, there were significant changes in the $b^{*}$ value of pink pigmented eggshells during incubation (Table 1). The value of $b^{*}$ was significantly different between day 0 and day 4 , with the analysis on day 4 showing more yellow and therefore an increase in $b^{*}$. This change may be due to the fumigation process (all samples except those for day 0 were treated) before incubation. We added the experiment of the pigment intensity test before and after formaldehyde fumigation. As a result, only $b^{*}$ was significantly different before and after formaldehyde fumigation (Table S1). As above, there was no significant difference in eggshell color during the incubation.

However, according to part 2 of the present study, the intensity of the pink pigment of the eggshell also influenced the spectral characteristics. Light pink pigment allowed more light to pass through the eggshell than did darker pigments. Among the eggshells with different pigment 
203

204

205

206

207

208

209

210

211

212

213

214

215

216

217

218

219

220

221

222

intensities, the LIP eggshells had the highest PT values. Therefore, according to the results of the

first two parts of the study, the incubation could cause the PT of the eggshells to decrease. Under

the influence of light, the development of embryos with pigmented shells is slower than for those

with unpigmented shells, and depigmentation of eggshells results in early hatching. Considering

that the lower the intensity of eggshell pigment, the higher the PT, the influence of the same light

intensity is different for different colors of egg, and our future research will focus on verifying

this conclusion.

incubation, being highest at the small pole, intermediate at the equator, and lowest at the large

pole. Pores are not uniformly distributed over the surface of the egg, and pore concentration

varies among the regions of the eggshell (Romanoff and Romanoff, 1949). Generally, the equator

and large pole have more pores than the small pole. However, the finding that the small pole of eggshell had higher PT than that of the large pole may be due to difference in the active pore area of the measured samples. A significant decrease in eggshell thickness was recorded on day 16. Theoretically speaking, the decrease of eggshell thickness may improve the PT. The slightly change before day 16 may cause no significantly PT change. The ranking order of shell components from outside to inside is shell, outer shell membrane and the inner shell membrane.

At the prophase (0-7 day during incubation), shell and membrane were combined closely. The combination was decreased during the incubation process. The membrane was almost separated from the shell on day 21. A micro-gap between the shell and membrane developed step-by-step 
223 during the incubation process. Reflection and scattering caused by the micro-gap may decrease

224 the light energy reaching the embryo, which was the reason of the decrease of PT on day 0-16.

225 The chicks were hatched on day 21 of incubation, and the eggshells were broken and slightly

226 destroyed, with the membrane and the shell completely separated from each other. These

227 phenomena resulted in significantly higher eggshell PT values on day 21 than on the other days.

228 The element analysis of the eggshells with the different pink pigment intensities showed

229 that the potassium content decreased significantly during incubation for eggshells with all

230 intensities of the pigment. $\mathrm{Na}^{+}$and $\mathrm{K}^{+}$are important ions to retain and regulate the cell potential

231 and osmotic pressure which are essential for living organisms. The embryo can only obtain $\mathrm{Na}^{+}$

232 and $\mathrm{K}^{+}$ions from the hatching egg itself. As parts of the egg, Shell and membrane may provide

$233 \mathrm{Na}^{+}$and $\mathrm{K}^{+}$ions to the embryo during incubation. This is the hypothesis for the mechanism of

234 changes in $\mathrm{Na}$ and $\mathrm{K}$ content during incubation.

235 In our study, the intensity of the pigment and the region of the eggshell influenced the PT of

236 the eggshell. Differences in the spectral characteristics of different eggshells may influence the

237 effects of photostimulation during the incubation of eggs.

\section{Conclusion}

239 In summary, the pigment intensity and the region of the eggshell influenced the PT of the

eggshell. Differences in the spectral characteristics of different eggshells may influence the

effects of photostimulation during the incubation of eggs. All of these results will be applicable

242 for perfecting the design of light intensity for lighted incubation to improve productivity.

\section{Acknowledgments}


244 This research was conducted at Zhejiang University. We gratefully acknowledge Huixian Chen 245 and Xiaoyu Liu (Zhejiang Guangda Breeding Poultry Corporation) for their help. We also thank 246 Chao Yuan (College of Animal Science, Zhejiang University) for his suggestions. 


\section{References}

Adam JH \& Dimond SJ. 1971. Influence of light on the time of hatching in the domestic chick. Animal Behaviour, 19: 226-229.

Archer G \& Mench J. 2014. The effects of the duration and onset of light stimulation during incubation on the behavior, plasma melatonin levels, and productivity of broiler chickens. Journal of Animal Science, 92: 1753-1758.

Bamelis F, Tona K, DE Baerdemaeker J \& Decuypere E. 2002. Detection of early embryonic development in chicken eggs using visible light transmission. British Poultry Science, 43: 204212.

Coleman MA \& Mcnabb RA. 1975. Photoacceleration of embryonic development in depigmented Japanese quail eggs. Poultry Science, 54: 1849-1855.

Fairchild B \& Christensen V. 2000. Photostimulation of turkey eggs accelerates hatching times without affecting hatchability, liver or heart growth, or glycogen content. Poultry Science, 79: 1627-1631.

Garwood V, Thornton E \& Lowe P. 1973. The effect of continuous illumination of incubating chicken eggs on embryonic development. Poultry Science, 52: 337-340.

Ghatpande, A., Ghatpande, S. \& Khan, M. 1994. Effect of different intensities of fluorescent light on the early development of chick embryos in ovo. Cellular and Molecular Biology Research, 41: 613-621.

Gold P \& Kalb J. 1976. Secondary heating of chicken eggs exposed to light during incubation. Poultry Science, 55: 34-39.

Lauber JK \& Shutze J. 1964. Accelerated growth of embryo chicks under the influence of light. Growth, 28: 179.

Maurer G, Portugal SJ \& Cassey P. 2011. Review: an embryo's eye view of avian eggshell pigmentation. Journal of Avian Biology, 42: 494-504.

Moreno J \& Osorno JL. 2003. Avian egg colour and sexual selection: does eggshell pigmentation reflect female condition and genetic quality? Ecology Letters, 6: 803-806.

Nelson NJ, Thompson MB, Pledger S, Keall SN \& Daugherty CH. 2004. Egg mass determines hatchling size, and incubation temperature influences post - hatching growth, of tuatara Sphenodon punctatus. Journal of Zoology, 263: 77-87.

Özkan S, Yalın S, Babacanoğlu E, Uysal S, Karadaş F \& Kozanoğlu H. 2012a. Photoperiodic lighting (16 hours of light: 8 hours of dark) programs during incubation: 2. Effects on early posthatching growth, blood physiology, and production performance in broiler chickens in relation to posthatching lighting programs. Poultry Science, 91: 2922-2930.

Özkan S, Yalın S, Babacanoğlu E, Kozanoğlu H, Karadaş F \& Uysal S. $2012 b$. Photoperiodic lighting (16 hours of light: 8 hours of dark) programs during incubation: 1 . Effects on growth and circadian physiological traits of embryos and early stress response of broiler chickens. Poultry Science, 91: 2912-2921.

Portugal S, Hauber M, Maurer G, Stokke B, Grim T \& Cassey P. 2014. Rapid development 
287

288

289

290

291

292

293

294

295

296

297

298

299

300

301

302

303

304

305

306

307

308

309

310

311

312

313

314

315

316

317

of brood - parasitic cuckoo embryos cannot be explained by increased gas exchange through the eggshell. Journal of Zoology, 293: 219-226.

Rogers LJ 2008. Development and function of lateralization in the avian brain. Brain Research Bulletin, 76: 235-244.

Romanoff AL \& Romanoff AJ. 1949. The avian egg. The avian egg.New York: John Wiley \& Sons, Inc.

Rozenboim I, Huisinga R, Halevy O \& EL Halawani M. 2003. Effect of embryonic photostimulation on the posthatch growth of turkey poults. Poultry Science, 82: 1181-1187.

Shafey T. 2004. Effect of lighted incubation on embryonic growth and hatchability performance of two strains of layer breeder eggs. British Poultry Science, 45: 223-229.

Shafey T \& Al-Mohsen T. 2002. Embryonic growth, hatching time and hatchability performance of meat breeder eggs incubated under continuous green light. Asian Australasian Journal of Animal Sciences, 15: 1702-1707.

Shafey T, AL-Batshan H, Ghannam M \& AL-Ayed M. 2005. Effect of intensity of eggshell pigment and illuminated incubation on hatchability of brown eggs. British Poultry Science, 46: 190-198.

Shafey T, AL-Mohsen T, AL-Sobayel A, AL-Hassan M \& Ghnnam M. 2002. Effects of eggshell pigmentation and egg size on the spectral properties and characteristics of eggshell of meat and layer breeder eggs. Asian Australasian Journal of Animal Sciences, 15: 297-302.

Tamimie H \& Fox M. 1967. Effect of continuous and intermittent light exposure on the embryonic development of chicken eggs. Comparative Biochemistry and Physiology, 20: 793799.

Walter J \& Voitle R. 1972. Effects of photoperiod during incubation on embryonic and postembryonic development of broilers. Poultry Science, 51: 1122-1126.

Zakaria A. 1989. Research note: Effect of fluorescent light on hatchability of commercial broiler parent stock eggs and on body weight of chickens hatched under large-scale commercial conditions. Poultry Science, 68: 1585-1587.

Zhang L, Zhang H, Qiao X, Yue H, Wu S, Yao J \& Qi G. 2012. Effect of monochromatic light stimuli during embryogenesis on muscular growth, chemical composition, and meat quality of breast muscle in male broilers. Poultry Science, 91: 1026-1031. 


\section{Table $\mathbf{1}$ (on next page)}

Table 1 . The $\triangle E, L^{*}, a^{*}, b^{*}$ value of eggshell during the incubation period $(0-21 d)$. 
1 Table 1. The $P$-values for the effect of time on the relative change of each color indicator $\left(\triangle \mathrm{E}, \mathrm{L}^{*}, \mathrm{a}^{*}, \mathrm{~b}^{*}\right)$.

2 The $\Delta E, L^{*}, a^{*}, b^{*}$ value of eggshell during the incubation period $(0-21 \mathrm{~d})$.

\begin{tabular}{|c|c|c|c|c|c|c|c|c|}
\hline \multicolumn{9}{|c|}{ Incubation period (day) } \\
\hline & & 0 & 4 & 8 & 12 & 16 & 21 & $P$ \\
\hline$\Delta \mathrm{E}$ & $24.9 \pm 1.0$ & $26.7 \pm 1.2$ & $25.2 \pm 1.0$ & $24.8 \pm 1.1$ & $25.5 \pm 1.2$ & $27.9 \pm 1.1$ & & $>0.05$ \\
\hline $\mathrm{L}^{*}$ & $76.3 \pm 0.8$ & $76.3 \pm 1.1$ & $77.4 \pm 0.8$ & $77.6 \pm 0.9$ & $77.5 \pm 0.9$ & $75.0 \pm 0.9$ & & $>0.05$ \\
\hline$a^{*}$ & $6.8 \pm 0.3$ & $6.8 \pm 0.6$ & $6.4 \pm 0.4$ & $6.3 \pm 0.5$ & $6.1 \pm 0.5$ & $6.2 \pm 0.4$ & & $>0.05$ \\
\hline$b^{*}$ & $18.9 \pm 0.6^{\mathbf{a}}$ & $21.7 \pm 0.6^{\mathbf{b}}$ & $20.6 \pm 0.6^{\mathrm{ab}}$ & $21.1 \pm 0.7^{\mathbf{b}}$ & $21.1 \pm 0.7^{\mathbf{b}}$ & $22.9 \pm 1.0^{\mathbf{b}}$ & & $<0.01$ \\
\hline
\end{tabular}

$3{ }^{1}$ Values are the mean \pm SEM, $\mathrm{n}=30$.

$4 \quad$ a, b, c, d means within a row followed by different superscripts are significantly different $(P<0.05)$.

5 


\section{Table 2 (on next page)}

Table 2. The element ( $\mathrm{K}, \mathrm{Na}, \mathrm{P}, \mathrm{Ca}$ and $\mathrm{Mg}$ ) content of eggshells with different intensities of the pink pigment of the eggshell during incubation. 
1 Table 2. The element ( $\mathrm{K}, \mathrm{Na}, \mathrm{P}, \mathrm{Ca}$ and $\mathrm{Mg}$ ) content ( $\mathrm{g} / 100 \mathrm{~g}$ of dry mass) of eggshells with different

2 intensities of the pink pigment of the eggshell during incubation.

\begin{tabular}{|c|c|c|c|c|c|c|c|c|}
\hline \multirow{2}{*}{$\begin{array}{l}\text { Intensity } \\
\text { pigment }\end{array}$} & \multirow{2}{*}{ Element } & \multicolumn{6}{|c|}{ Incubation period (d) } & \multirow[b]{2}{*}{$P$ value } \\
\hline & & 0 & 4 & 8 & 12 & 16 & 21 & \\
\hline \multirow{5}{*}{$\mathrm{LIP}^{1}$} & $\mathrm{Na}$ & $0.099 \pm 0.006$ & $0.101 \pm 0.006$ & $0.098 \pm 0.015$ & $0.106 \pm 0.008$ & $0.098 \pm 0.002$ & $0.086 \pm 0.01$ & $>0.05$ \\
\hline & $\mathrm{P}$ & $0.137 \pm 0.005$ & $0.143 \pm 0.019$ & $0.144 \pm 0.011$ & $0.142 \pm 0.011$ & $0.157 \pm 0.032$ & $0.156 \pm 0.026$ & $>0.05$ \\
\hline & $\mathrm{Ca}$ & $39.004 \pm 0.805$ & $37.954 \pm 0.734$ & $37.759 \pm 0.635$ & $38.219 \pm 0.524$ & $38.209 \pm 0.19$ & $37.863 \pm 0.608$ & $>0.05$ \\
\hline & $\mathrm{K}$ & $0.047 \pm 0.001$ & $0.051 \pm 0.006$ & $0.041 \pm 0.005$ & $0.045 \pm 0.003$ & $0.046 \pm 0.003$ & $0.038 \pm 0.004$ & $<0.05$ \\
\hline & $\mathrm{Mg}$ & $0.454 \pm 0.043$ & $0.465 \pm 0.04$ & $0.454 \pm 0.052$ & $0.507 \pm 0.031$ & $0.492 \pm 0.037$ & $0.432 \pm 0.039$ & $>0.05$ \\
\hline \multirow{5}{*}{$\mathrm{MIP}^{1}$} & $\mathrm{Na}$ & $0.11 \pm 0.008$ & $0.104 \pm 0.004$ & $0.107 \pm 0.008$ & $0.102 \pm 0.012$ & $0.09 \pm 0.004$ & $0.091 \pm 0.004$ & $<0.05$ \\
\hline & $\mathrm{P}$ & $0.122 \pm 0.022$ & $0.143 \pm 0.004$ & $0.157 \pm 0.006$ & $0.153 \pm 0.011$ & $0.154 \pm 0.021$ & $0.159 \pm 0.038$ & $>0.05$ \\
\hline & $\mathrm{Ca}$ & $40.929 \pm 1.963$ & $38.262 \pm 0.359$ & $39.438 \pm 3.033$ & $37.301 \pm 0.559$ & $37.856 \pm 0.292$ & $38.342 \pm 0.564$ & $>0.05$ \\
\hline & $\mathrm{K}$ & $0.048 \pm 0.002$ & $0.051 \pm 0.003$ & $0.048 \pm 0.007$ & $0.043 \pm 0.003$ & $0.041 \pm 0.002$ & $0.04 \pm 0.002$ & $<0.01$ \\
\hline & $\mathrm{Mg}$ & $0.424 \pm 0.042$ & $0.451 \pm 0.006$ & $0.499 \pm 0.05$ & $0.456 \pm 0.023$ & $0.442 \pm 0.041$ & $0.447 \pm 0.026$ & $>0.05$ \\
\hline \multirow{5}{*}{$\mathrm{DIP}^{1}$} & $\mathrm{Na}$ & $0.101 \pm 0.014$ & $0.094 \pm 0.003$ & $0.096 \pm 0.002$ & $0.09 \pm 0.001$ & $0.093 \pm 0.005$ & $0.091 \pm 0.008$ & $>0.05$ \\
\hline & $\mathrm{P}$ & $0.136 \pm 0.007$ & $0.15 \pm 0.008$ & $0.14 \pm 0.006$ & $0.144 \pm 0.019$ & $0.145 \pm 0.024$ & $0.14 \pm 0.03$ & $>0.05$ \\
\hline & $\mathrm{Ca}$ & $39.186 \pm 0.876$ & $38.487 \pm 0.128$ & $37.994 \pm 0.404$ & $37.822 \pm 1.215$ & $37.814 \pm 0.258$ & $38.322 \pm 0.215$ & $>0.05$ \\
\hline & $\mathrm{K}$ & $0.047 \pm 0.003$ & $0.048 \pm 0.004$ & $0.043 \pm 0.004$ & $0.037 \pm 0.004$ & $0.043 \pm 0.005$ & $0.038 \pm 0.005$ & $<0.05$ \\
\hline & $\mathrm{Mg}$ & $0.455 \pm 0.049$ & $0.44 \pm 0.021$ & $0.446 \pm 0.05$ & $0.37 \pm 0.01$ & $0.465 \pm 0.044$ & $0.429 \pm 0.045$ & $>0.05$ \\
\hline
\end{tabular}

$3{ }^{1} \mathrm{LIP}=$ light intensity pigment, $\mathrm{MIP}=$ medium intensity pigment, and $\mathrm{DIP}=$ dark intensity pigment

$4{ }^{2}$ Values are the mean \pm SEM.

5 


\section{Figure 1 (on next page)}

Figure 1. The schematic of the detection equipment for light transmission. 


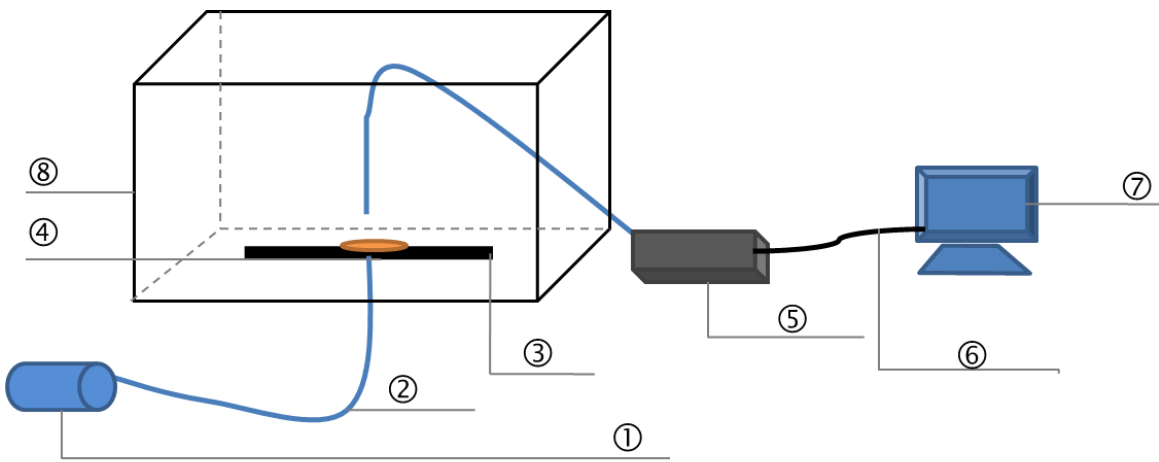

(1) Light source

(2) Optical fiber

(3) Sample holder

(4) Sample(eggshell)

(5) Spectrometer

(6) Data line

(7) Computer

(8) Black light box 


\section{Figure 2 (on next page)}

Figure 2. The PT of light over the wavelength range of 380 to $780 \mathrm{~nm}$ at the small pole, the equator and the large pole of pink pigment eggshells on days 0 during incubation. $S$ $=$ small pole, $\mathrm{E}=$ equator and $\mathrm{L}=$ large pole.

(a) shows the PT of light over the wavelength range of 380 to $780 \mathrm{~nm}$ at the small pole, the equator and the large pole of pink pigment eggshells on days 0 during incubation. (b) shows the mean PT value of eggshell over the wavelength range of 380 to $780 \mathrm{~nm}$. 


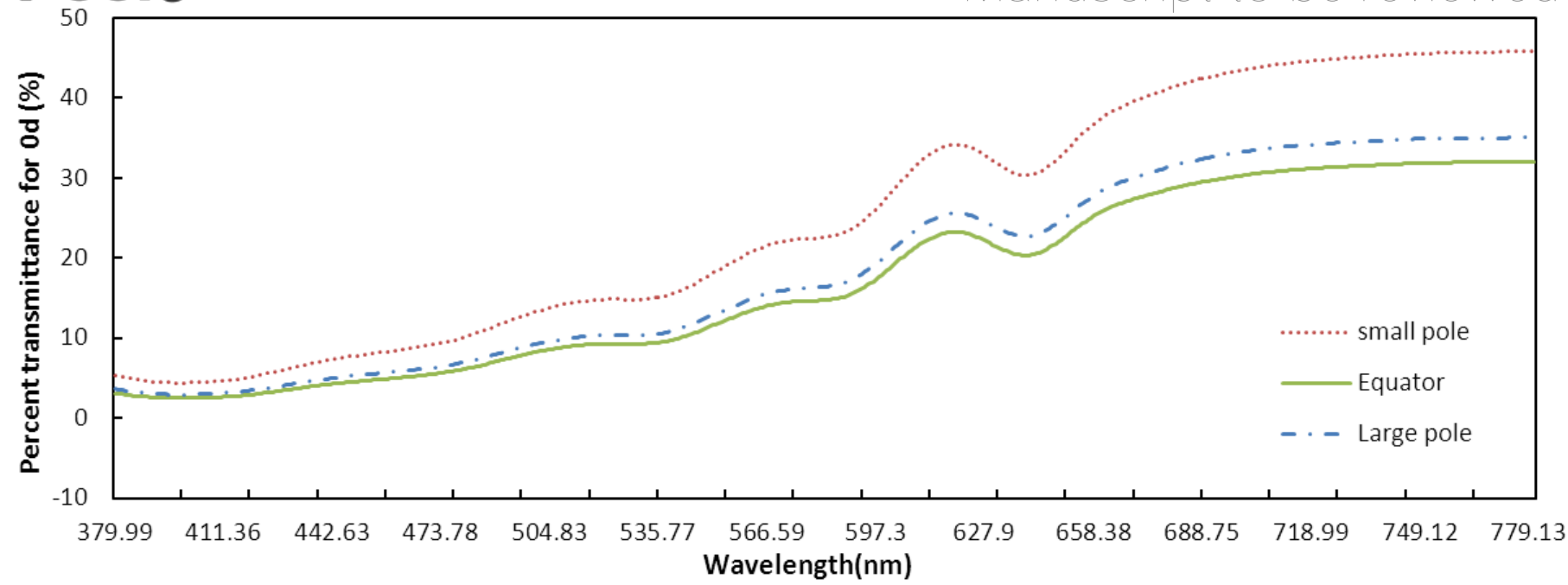

(a)

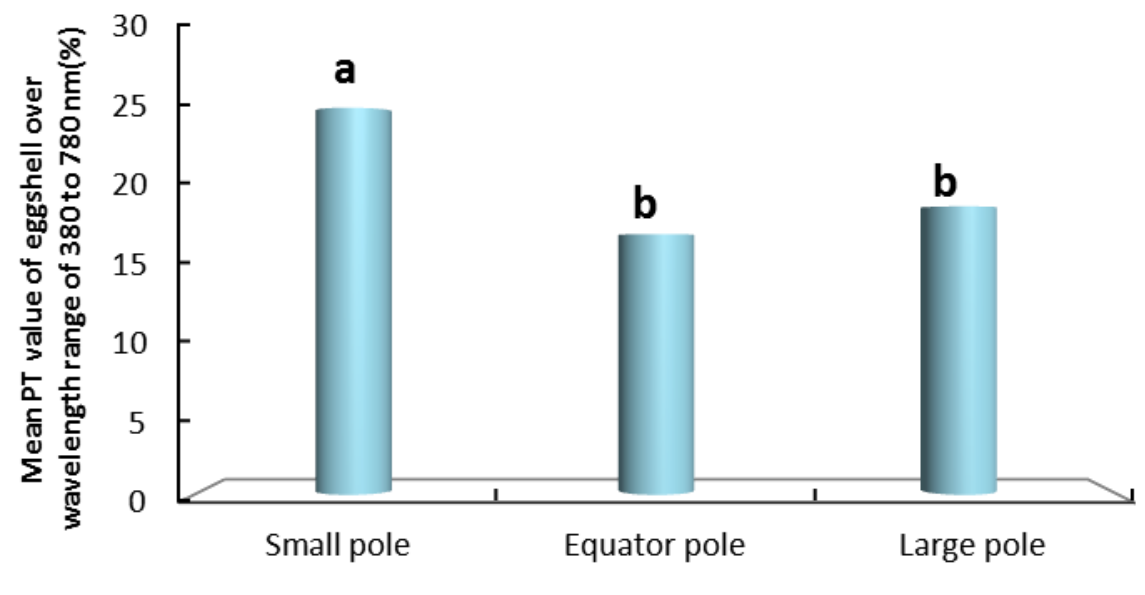

Different regions of eggshell on $0 \mathrm{~d}$ during incuabion

(b) 


\section{Figure 3 (on next page)}

Figure 3. The PT of light over the wavelength range of 380 to $780 \mathrm{~nm}$ for the light, middle and dark levels of pink pigment in eggshells on day 0 during the incubation. LIP $(L)=$ light intensity pigment, $M I P(M)=$ medium intensity pigment and $D I P(D)=$ dark

(a) shows the PT of light over the wavelength range of 380 to $780 \mathrm{~nm}$ for the light, middle and dark levels of pink pigment in eggshells on day 0 during the incubation. (b) shows the mean PT value of eggshell over the wavelength range of 380 to $780 \mathrm{~nm} .<$ ?xml:namespace prefix = o ns = "urn:schemas-microsoftcom:office:office" /> 


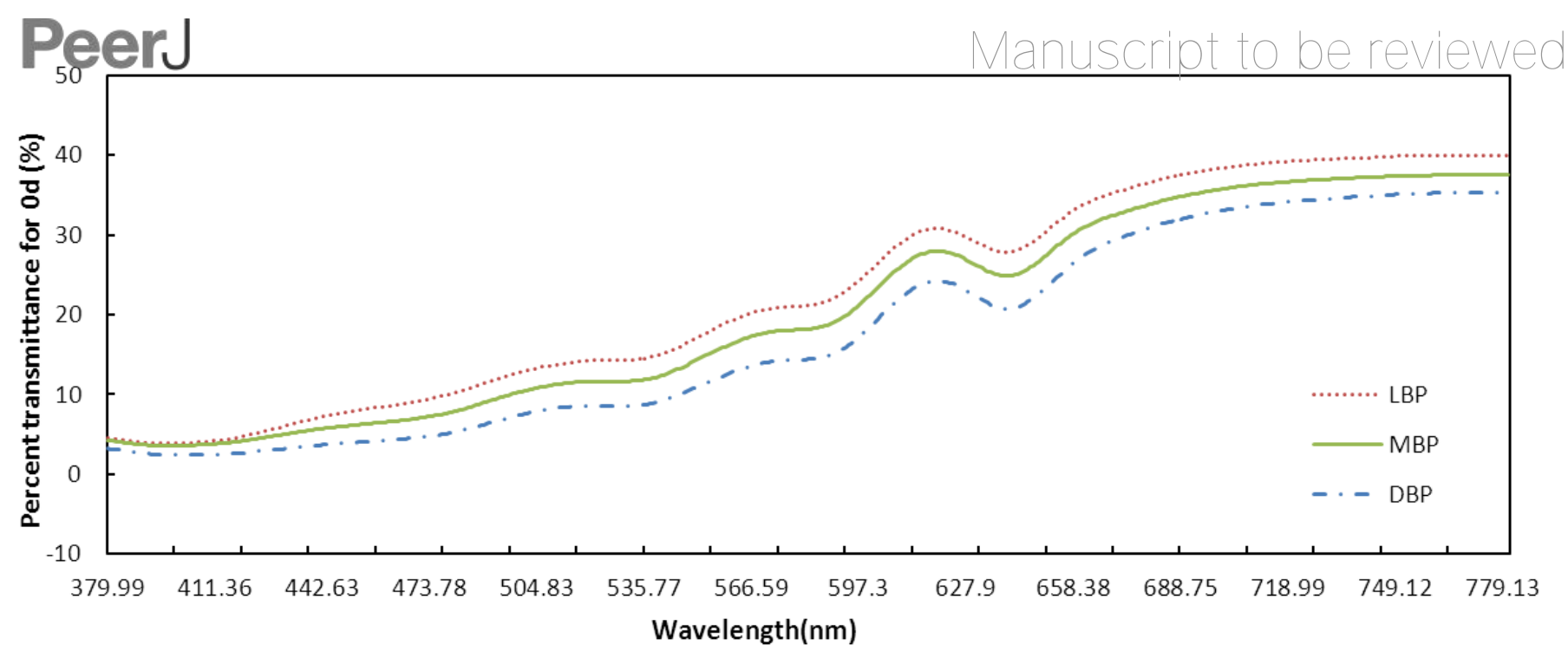

(a)

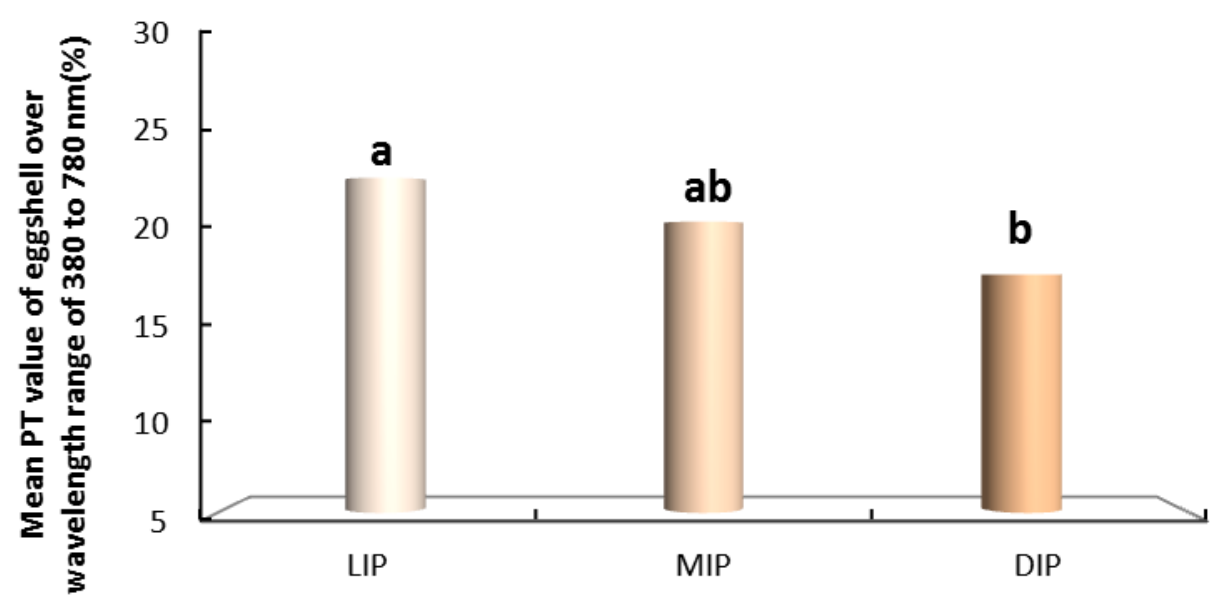

Eggsell with different pigment intensities on $0 \mathrm{~d}$ during incubation

(b) 
Figure 4 (on next page)

Figure 4. The PT(\%) of light over the wavelengths of 380 to $780 \mathrm{~nm}$ at different regions of the eggshell and at different levels of intensity of the pink pigment of the eggshells at different periods during incubation. Values are the mean $\pm S E M, n=30$. 


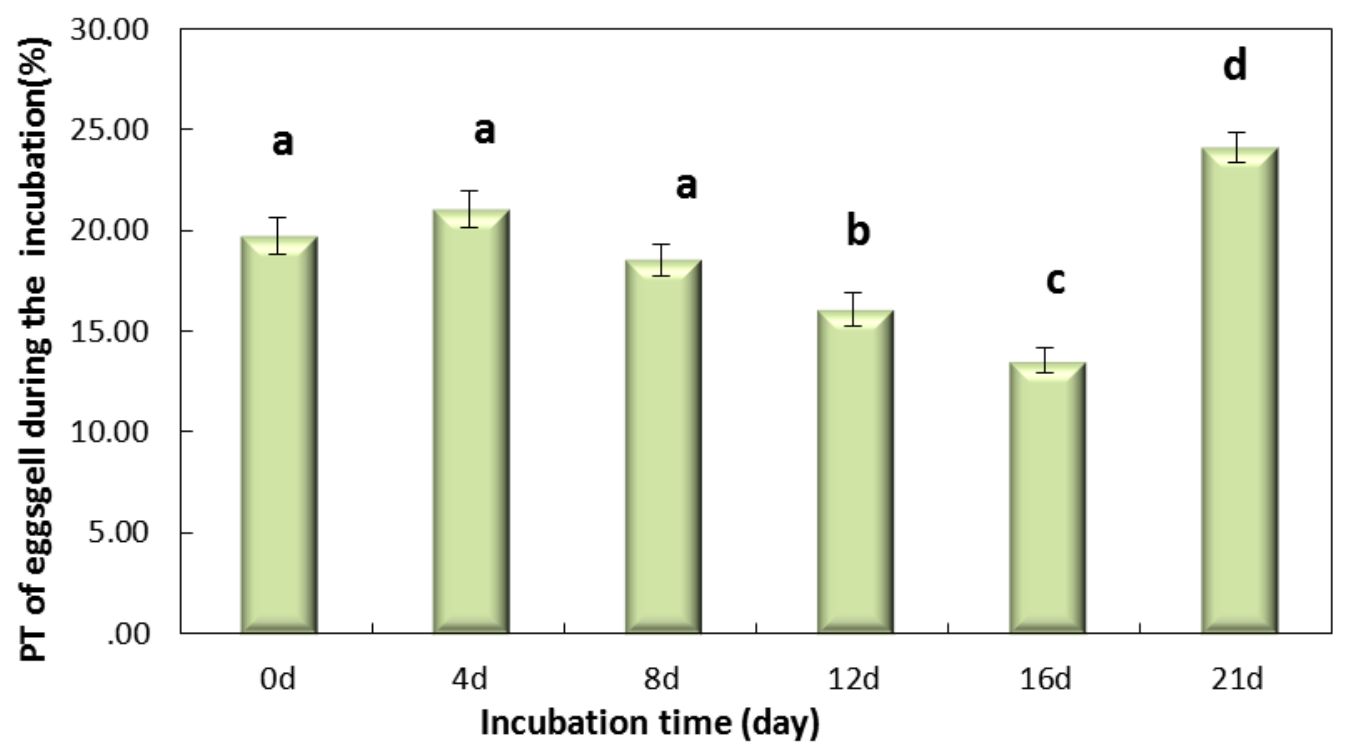


Figure $\mathbf{5}$ (on next page)

Figure 5. The thickness of eggshell on days $0,4,8,12,16$ and 21 during the incubation. And if followed by different superscripts, values are significantly different $(P \square 0.05)$. Data were presented as mean \pm SEM, $n=30$. 


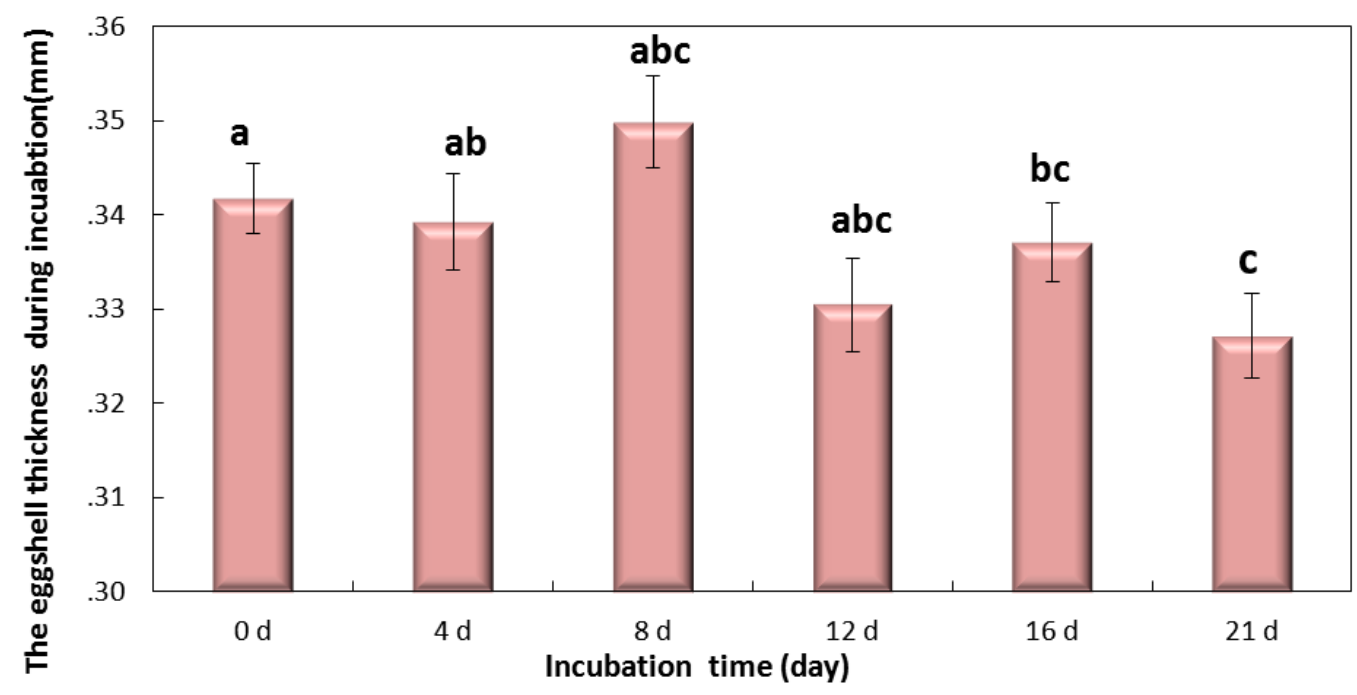

